First publ. in: Physical Review E 78 (2008), 2, 6106

\title{
Nonlocal elastic compliance for soft solids: Theory, simulations, and experiments
}

\author{
K. Franzrahe, P. Keim, G. Maret, and P. Nielaba \\ Fachbereich Physik, Universität Konstanz, Postfach M692, 78457 Konstanz, Germany
}

S. Sengupta

Satyendra Nath Bose National Centre for Basic Sciences, Block-JD, Sector-III, Salt Lake, Kolkata 700 098, India

\begin{abstract}
The nonlocal elastic response function is crucial for understanding many properties of soft solids. This may be obtained by measuring strain-strain autocorrelation functions. We use computer simulations as well as video microscopy data of superparamagnetic colloids to obtain these correlations for two-dimensional triangular solids. Elastic constants and elastic correlation lengths are extracted by analyzing the correlation functions. We show that to explain our observations displacement fluctuations in a soft solid need to contain affine (strain) as well as nonaffine components.
\end{abstract}

Elasticity theory $[1,2]$ is based on the assumption that the displacement field $\mathbf{u}(\mathbf{r})$ is smooth and continuous for all positions $\mathbf{r}$ within a volume $V$. It is expected that this assumption breaks down [3] below a limiting length $\xi_{\mathrm{el}}$. How small is $\xi_{\text {el }}$ in practice, and what are the consequences of the breakdown? What implications does this have on the mechanical response of the material? While for most conventional engineering problems such issues are practically irrelevant, they do become important when investigating microelastic and microrheological properties of soft solids [6]. We answer these questions in the context of the nonlocal mechanical response of a soft solid in two dimensions (2D). Nonlocal elastic effects are important whenever strain gradients are large, e.g., near crack tips, grain boundaries, and interfaces [4]. They should also be important if the linear dimension of the solid is not much larger than $\xi_{\mathrm{el}}$. Both of these criteria are satisfied in colloidal solids under usual experimental conditions [5].

Recently, there has been a lot of interest in obtaining the elastic moduli of soft solids [6,7] from fluctuations of particle coordinates [8-12]. These methods have several advantages. First, no specialized equipment is necessary other than standard video microscopy [7]. Second, no external forces need to be applied which may change the very properties that are being measured. This is especially convenient when trying to obtain elastic constants near the dislocation unbinding transition [1] where the solid is sensitive to external perturbations [11]. Lastly, these techniques are general enough so that they may be applied to a wide variety of systems [13]. Here we extend this procedure a step further to study nonlocal [4] elasticity for solids in two dimensions far from the melting transition.

The nonlocal elastic response function, or compliance $\chi_{i j}\left(\mathbf{r}, \mathbf{r}^{\prime}\right)(i=x, y)$, is defined as the strain $\varepsilon_{i j}\left(\mathbf{r}^{\prime}\right)$ produced at position $\mathbf{r}^{\prime}$ due to a stress $\sigma_{i j}(\mathbf{r})$ at $\mathbf{r}$. This is given by $\chi_{i j}=\left(k_{B} T\right)^{-1} G_{i j}$, where, for a homogeneous solid, $G_{i j}\left(\mathbf{r}^{\prime}\right)$ $=\left\langle\varepsilon_{i}(\mathbf{0}) \varepsilon_{j}\left(\mathbf{r}^{\prime}\right)\right\rangle$ is the strain-strain correlation function. The $\langle\cdots\rangle$ denote a thermal average (and one over the choice of origin) and $k_{B} T$ is the Boltzmann constant times the temperature [1]. The linear combinations of components of the strain tensor $\varepsilon$ relevant to a $2 \mathrm{D}$ solid are $e_{i}(i=1,2,3)$ defined as derivatives of the displacement field $\mathbf{u}(x, y)$ calculated from the undistorted reference lattice and given by $e_{1}=\partial u_{x} / \partial x$ $+\partial u_{y} / \partial y$ (volume), $e_{2}=\partial u_{x} / \partial x-\partial u_{y} / \partial y$ (deviatoric), and $e_{3}$ $=\left(\partial u_{x} / \partial y+\partial u_{y} / \partial x\right) / 2$ (shear). For a solid at $T \neq 0$ these are fluctuating quantities, the width of the distribution being fixed by the relevant elastic constant [8,9]. In classical, linear elasticity strain fluctuations represent affine deformations, i.e., the particle positions are obtainable, at all times, from the ideal $T=0$ reference lattice position by local affine transformations. These do not, however, exhaust all possible low energy excitations of a real solid [14].

Nevertheless, we begin with a strain-only derivation of $G_{i j}$ for a fluctuating solid using (nonlocal) elasticity. We use the dimensionless elastic free energy functional, $\mathcal{F}$ $=\frac{k_{B} T}{a^{2}} \frac{1}{2} \int d \mathbf{r} \Sigma_{i}\left[a_{i} e_{i}^{2}+c_{i}\left(\nabla e_{i}\right)^{2}+c_{i}^{\prime}\left(\nabla^{2} e_{i}\right)^{2}\right]$. Energy and length scales are set by $k_{B} T$ and lattice parameter $a$, respectively. The parameters $a_{i}(i=1-3)$ are the elastic constants and $c_{i}$ and $c_{i}^{\prime}$ set the length scales over which strain fluctuations decay. The constraint $\boldsymbol{\nabla} \times(\boldsymbol{\nabla} \times \boldsymbol{\epsilon})^{T}=0$ (viz., St. Venant's condition [15]) ensures that fluctuations of the particle positions are locally affine and strains can be defined everywhere, i.e., $\mathbf{u}(\mathbf{r})$ is smooth everywhere. In addition, the strains need to ensure mechanical stability, viz., $\partial \sigma_{i j} / \partial x_{j}=0$ where $\sigma_{i j}$ $=\delta \mathcal{F} / \delta \epsilon_{i j}$ are the conjugate stresses. In Fourier space these relations lead to the following equations connecting strain components in equilibrium: $\widetilde{e}_{i}=\widetilde{\mathcal{Q}}_{i j}(\mathbf{k}) \widetilde{e}_{j}$, where the kernels $\left.\widetilde{\mathcal{Q}}_{i j}(\mathbf{k})\right|_{|k| \neq 0}$ are explicitly given as $\widetilde{\mathcal{Q}}_{12}=\left(a_{3}-2 a_{2}\right) /\left(2 a_{1}\right.$ $\left.+a_{3}\right)\left(k_{x}^{2}-k_{y}^{2}\right) / k^{2}, \quad \widetilde{\mathcal{Q}}_{13}=\left(4 a_{2}-2 a_{3}\right) /\left(a_{1}+a_{2}\right)\left(k_{x} k_{y}\right) /\left(k^{2}\right)$, and $\widetilde{\mathcal{Q}}_{23}=-\left(4 a_{1}+2 a_{3}\right) /\left(a_{1}+a_{2}\right)\left(k_{x} k_{y}\right) /\left(k_{x}^{2}-k_{y}^{2}\right)$. Note that $\widetilde{\mathcal{Q}}_{i j}(0)$ $=0$ - uniform strains are independent. These relations may now be used to eliminate any two of the strains and obtain a free energy functional only in terms of the single remaining independent strain. We therefore get immediately from equipartition,

$$
\begin{gathered}
\widetilde{G}_{i i}(\mathbf{k} \neq \mathbf{0})^{-1}=\left(a_{i}+c_{i} k^{2}+c_{i}^{\prime} k^{4}\right. \\
\left.+\sum_{j \neq i}\left(a_{j}+c_{j} k^{2}+c_{j}^{\prime} k^{4}\right)\left[\widetilde{\mathcal{Q}}_{j i}(\mathbf{k})\right]^{2}\right) \\
\widetilde{G}_{i i}(0)^{-1}=a_{i} .
\end{gathered}
$$


a)

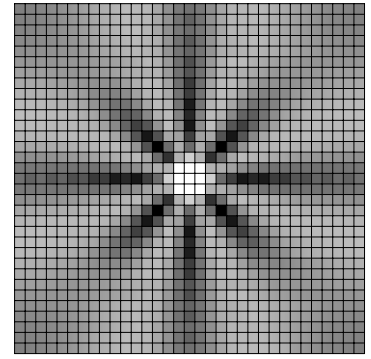

c)

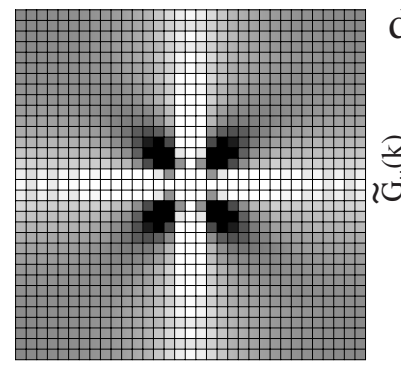

b)

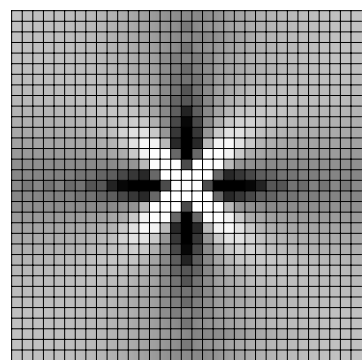

d)

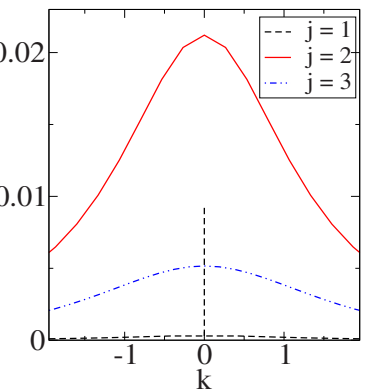

FIG. 1. (Color online) Analytic strain-strain correlation functions for a triangular lattice with parameters obtained from fits to simulations: subfigures show real space density plots of the strainstrain correlation functions over a box of size $L=34.35 a$ : (a) $(L / a)^{2} G_{11} \quad$ (gray scale black to white $\left.[-0.003,0.015]\right)$, (b) $(L / a)^{2} G_{22}([-0.1,0.1])$, and (c) $(L / a)^{2} G_{33}([-0.03,0.03])$. (d) Cuts of $\widetilde{G}_{j j}$ along various directions in $k$ space: $k_{x}=k_{y}$ for $j=2, k_{x}=0$ for $j=3$, and $k_{y}=2 k_{x}$ for $j=1$. Note the discontinuity in $\widetilde{G}_{11}$ at $k=0$.

Consider the shape of $\widetilde{G}_{i i}(\mathbf{k})$ implied by (1). Along most directions in $k$ space all the correlation functions are discontinuous, i.e., $\lim _{k \rightarrow 0} \widetilde{G}_{i i}(k) \neq \widetilde{G}_{i i}(0)$ —an intrinsic property of $\widetilde{G}_{i i}(\mathbf{k})$. If the solid is completely describable within the framework of classical, linear elasticity, this should be observable in simulations and experiments. $G_{22}$ is smooth and continuous along the direction $k_{x}= \pm k_{y}$ as is $G_{33}$ along $k_{x}$ $=0$ or $k_{y}=0$. In these special directions the coupling terms involving $\widetilde{\mathcal{Q}}_{i j}(\mathbf{k})$ vanish and the correlation functions are continuous, smoothly extrapolating to $k=0$. Therefore these directions can be used in order to extract the elastic constants and correlation lengths. The correlation functions $G_{i i}, i$ $=1-3$, are plotted in Figs. 1(a) $-1(\mathrm{c})$ in real space. In Fig. 1(d) we show cuts through these correlation functions in $k$ space showing especially the discontinuity in $\widetilde{G}_{11}$. The rotational symmetry of $G_{11}$ is eightfold, while the other two correlation functions show a fourfold rotational symmetry. These symmetries are inherent and independent of the symmetry of the analyzed solid.

What do these correlation functions look like in soft matter solids as, e.g., colloidal crystals? Experiments demonstrate [10] that colloidal crystals far from the melting transition are harmonic. It is instructive, therefore, to compare these analytic predictions for $G_{i i}$ first with Monte Carlo simulations [17] of a harmonic triangular solid of $N$ particles with a Hamiltonian $\mathcal{H}=k_{B} T(f / 2) \sum_{m, n=1}^{N}\left(\mid \mathbf{r}_{m}-\mathbf{r}_{n}-a\right)^{2}$ where $f$ is the spring constant and $a$ is the lattice parameter. Energy and length scales are set by $k_{B} T$ and $a$, respectively. The elastic constants are given in terms of $f$ by $a_{1}=\sqrt{3} / 2 f=K$, the

a)
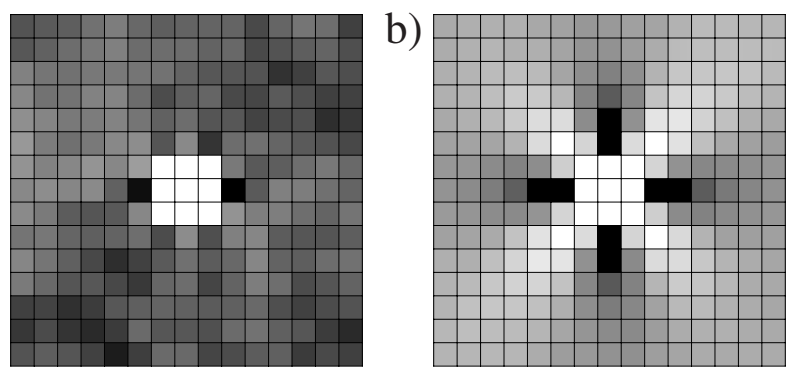

c)
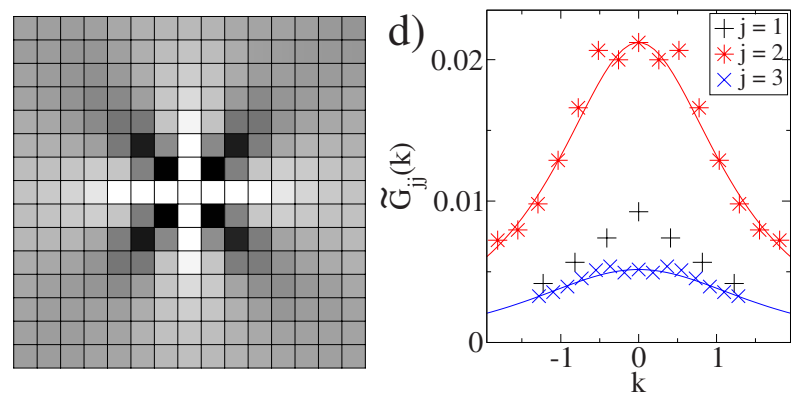

FIG. 2. (Color online) Simulation of a harmonic triangular lattice. Subfigures show real space density plots of the strain-strain correlation functions over a box of size $L=34.35 a$. (a) $(L / a)^{2} G_{11}$ (gray scale black to white $[-0.003,0.015])$, (b) $(L / a)^{2} G_{22}$ $([-0.1,0.1])$, and (c) $(L / a)^{2} G_{33}([-0.03,0.03])$. (d) Simulation data (symbols) and fits (lines) of $\widetilde{G}_{j j}$ along various directions in $k$ space: $k_{x}=k_{y}$ for $j=2, k_{x}=0$ for $j=3$, and $k_{y}=2 k_{x}$ for $j=1$.

bulk modulus, and $a_{2}=\sqrt{3} / 4 f=\mu$ and $a_{3}=\sqrt{3} f=4 \mu$, where $\mu$ is the shear modulus. The relation $a_{3}=4 a_{2}$ signifies an isotropic solid [1] in 2D, and $a_{1}=2 a_{2}$ is the so-called Cauchy condition $[2,8]$. Strains are calculated with respect to the average particle positions $\left\{\mathbf{r}_{n}(0)\right\}$ as a reference (corrected for center of mass motion and rotations of the system) using the scheme of Falk and Langer [16].

Briefly, we obtain the "best fit" local affine strain $\epsilon_{i j}$ which maps as nearly as possible all the particles $n$ in the immediate neighborhood $\Omega$ of a tagged particle from the reference to the transformed lattice. This is done by minimizing the (positive) scalar quantity,

$D^{2}(\mathbf{r}, t)={ }_{n \in \Omega \quad i}\left(r_{n}^{i}-r_{0}^{i}-{ }_{j}\left(\delta_{i j}+\epsilon_{i j}\right)\left[r_{n}^{j}(0)-r_{0}^{j}(0)\right]^{2}\right.$,

with respect to choices of affine $\epsilon_{i j}$. Here $i, j=x, y$ and $r_{n}^{i}(0)$ and $r_{n}^{i}$ are the $i$ th component of the position vector of the $n$th particle in the reference and transformed lattices, respectively. The local strains, thus obtained, are averaged within blocks of a size much smaller than the system size $L$ to obtain coarse-grained strains from which correlation functions are computed.

Our results for simulations of a harmonic triangular lattice with $N=5822, f=200 / \sqrt{3}$, lattice parameter $a=(2 / \sqrt{3})^{1 / 2}$, and $k_{B} T=1$ are shown in Figs. 2(a)-2(d). The theoretical values for the elastic constants in units of $k_{B} T / a^{2}$ are $a_{1}=100, a_{2}$ $=50$, and $a_{3}=200$. A standard Metropolis algorithm with open boundary conditions was used [17]. After $3 \times 10^{6}$ Monte Carlo steps for equilibration, configurations are stored every $10^{3}$ Monte Carlo steps. A total of $36 \times 10^{3}$ configurations is used for the subsequent analysis. We obtain $a_{1}$ 
$=108.16, a_{2}=47.14$, and $a_{3}=193.80$ to less than $8.2 \%$ numerical uncertainty; most of the errors arising from the fitting procedures and numerical Fourier transforms. Note that the symmetry requirement for the triangular lattice, $a_{2}$ $=a_{3} / 4$, is satisfied to within $2.8 \%$. More accurate estimates (to within $2.5 \%$ ) of the elastic constants can be obtained from finite size scaling of the probability distributions for the strains [9]. The quantities $c_{2}=27.80, c_{2}^{\prime}=0.833, c_{3}=63.16$, and $c_{3}^{\prime}=3.43$ imply an average elastic correlation length $\xi_{\text {el }}$ $\sim \sqrt{c_{i}} \sim 5-8$ lattice spacings below which local linear elasticity breaks down. The overall shapes of the $G_{i i}$ agree well with the strain-only expression in Eq. (1), the spatial anisotropy being reproduced quite faithfully [Figs. 2(a)-2(c)]. In contrast, when we compare the discontinuities in $\widetilde{G}_{i i}(k)$, the data differ spectacularly from the strain-only values. This is most obvious in the $k$ space cuts for $\widetilde{G}_{11}(k)$ [Figs. $1(\mathrm{~d})$ and $2(\mathrm{~d})]$. For $k \rightarrow 0(k \neq 0) \widetilde{G}_{11}(k)$ is 25 times larger than predicted, so the harmonic system is almost 25 times softer for $k \neq 0$ than the analytic predictions - well beyond random errors in the data.

Is this also the case in a real colloidal crystal? The experimental system studied is an improved version of Ref. [9]. Spherical colloids (diameter $d=4.5 \mu \mathrm{m}$ ) are confined by gravity to a water/air interface formed by a hanging water droplet. The field of view was $835 \times 620 \mu \mathrm{m}^{2}$, containing typically $2 \times 10^{3}$ particles whereas the whole system has a size of $50 \mathrm{~mm}^{2}$ and contains about $3 \times 10^{5}$ particles. The analyzed volume $V$ contains 840 particles arranged in a triangular lattice. The particles are superparamagnetic. Therefore a magnetic field $\vec{H}$ applied perpendicularly to the water/ air interface induces a magnetic moment $\vec{M}=\chi_{M} \vec{H}$ in each particle. This leads to a repulsive (inverse cubic) dipoledipole pair interaction with the dimensionless interaction strength given by $\Gamma$, the ratio of potential to thermal energy. Thus $\Gamma$ can be interpreted as an inverse temperature. The coordinates of all particles at each time step are analyzed and the trajectories are recorded for 2-3 h. The local strains and the strain correlation functions are obtained from these trajectories using the same techniques described above for the simulation data, except that now we need to account for the medium surrounding the analyzed volume $V$ which leads to a coupling between volume and shear fluctuations. In the limit that $V$ is far smaller than the complete system $G_{11}(0)=a_{1}$ $=K+\mu$ and $\mu$ can be extracted from pure shear fluctuations in the embedded system, i.e., fluctuations of $2 \theta=\left(\partial u_{y} / \partial x\right.$ $\left.-\partial u_{x} / \partial y\right)$. The shear fluctuations $e_{2}, e_{3}$, and $2 \theta$ are coupled and the kernel relating $\tilde{e}_{3}$ and $2 \tilde{\theta}$ is $\tilde{Q}_{32 \theta}=\frac{1}{2}\left(k_{x}^{4}-k_{y}^{4}\right) /\left[\left(k_{x}^{2}\right.\right.$ $\left.\left.-k_{y}^{2}\right)^{2}+k_{x}^{2} k^{2}\left(\left(4 a_{1}+2 a_{3}\right) /\left(a_{1}+a_{2}\right)\right)\right]$ [18]. The free energy of the embedded system can be written as a functional of $2 \theta$ and we obtain the correlation function

$$
\begin{aligned}
\widetilde{G}_{2 \theta 2 \theta}(\mathbf{k} \neq \mathbf{0})^{-1}= & \left(a_{3}+c_{3} k^{2}+c_{3}^{\prime} k^{4}+{ }_{j=1}^{2}\left(a_{j}+c_{j} k^{2}+c_{j}^{\prime} k^{4}\right)\right. \\
& \times\left[\widetilde{\mathcal{Q}}_{j 3}(\mathbf{k})\right]^{2}\left[\widetilde{\mathcal{Q}}_{32 \theta}(\mathbf{k})\right]^{2}, \\
& \widetilde{G}_{2 \theta 2 \theta}(0)^{-1}=a_{3} / 4 .
\end{aligned}
$$

a)

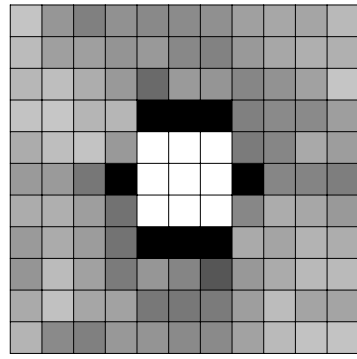

c)

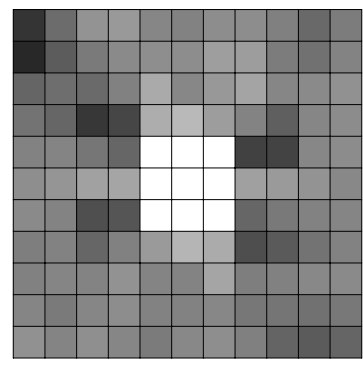

b)
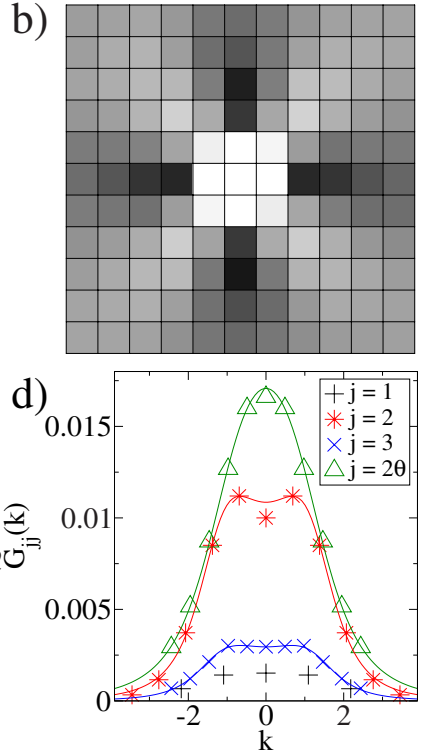

FIG. 3. (Color online) Colloidal crystal at $\Gamma=150$ [19]. The strain correlation functions were obtained from 3600 independent configurations for a defect-free square region $(L=12.881 a)$. (a)-(c) show real space strain-strain correlation functions $(L / a)^{2} G_{11}$ (gray scale, black to white $[-0.0015,0.0015]),(L / a)^{2} G_{22}$ $([-0.05,0.075])$, and $(L / a)^{2} G_{2 \theta 2 \theta}([-0.02,0.04])$. (d) Data (symbols) and fits (lines) of $\widetilde{G}_{j j}$ along various directions in $k$ space: $k_{x}$ $=k_{y}$ for $j=2, k_{x}=0$ for $j=3$ and $j=2 \theta$, and $k_{y}=2 k_{x}$ for $j=1$.

The behavior of the strain correlation functions of the colloidal crystal shown in Fig. 3 is similar to that of the harmonic system (Fig. 2). The anisotropy of the correlation functions agrees well with theory and simulation results. Elastic constants in units of $k_{B} T / a^{2}$ obtained from the $k=0$ values of $G_{11}(K=598.17)$ and $G_{2 \theta 2 \theta}(\mu=60.20)$ lie within $2.9 \%$ of those obtained by analyzing the same data using the procedure of Ref. [9]. For the superparamagnetic colloidal crystal $\mu=K / 10$. This relation is recovered within less than $1.0 \%$. The symmetry of $G_{22}$ is recovered well, which is a sign that defects lying outside the analyzed region have no impact on the analysis. Again the discontinuities in $\widetilde{G}_{i i}(k)$ are much reduced compared to the strain-only values, as was the case in the simulations. In both cases the correlations seem to acquire an additional, isotropic contribution, the origin of which is unclear within our strain-only model.

The residual value of $D^{2}$ in Eq. (2) is a measure of nonaffineness, i.e., failure to obtain a unique value for displacement derivatives $\epsilon_{i j}$. Even the harmonic solid, which conserves local topology, has spontaneous nonaffine fluctuations of zero mean. We have plotted the correlation function corresponding to $D^{2}$ for the colloidal solid [Fig. 4(a)] and the harmonic system [Fig. 4(b)]. Both depict an isotropic function decaying rapidly within a range $\sim \xi_{\mathrm{el}}$. Small numerical errors account for at most a small overall approximately constant offset in $G_{D^{2}}$. In Fig. 4(c) we plot the probability distribution of $D^{2}$ for the colloidal crystal and for harmonic solids with $f=200 / \sqrt{3}$ and $1200 / \sqrt{3}$. The colloidal crystal has an intermediate stiffness, compared to the two harmonic systems. We observe that stiffer solids generate smaller $D^{2}$, showing that its origin is thermal. This short-wavelength, 

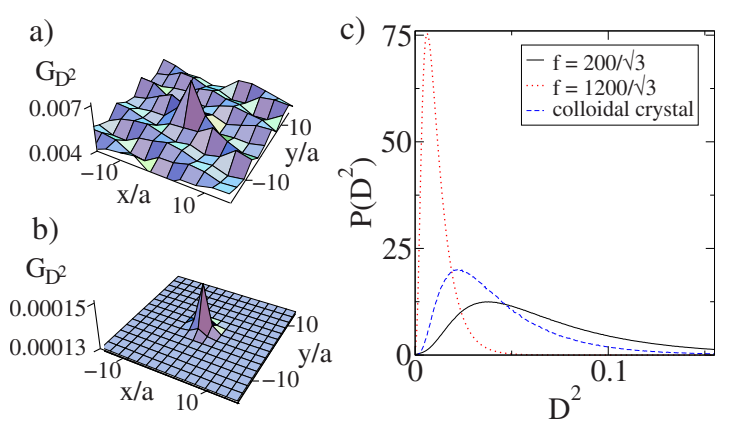

FIG. 4. (Color online) (a) Correlation function of the nonaffine parameter $G_{D^{2}}(x, y)$ for the colloidal crystal at $\Gamma=150$ [19]. (b) Correlation function $G_{D^{2}}(x, y)$ for the harmonic system $(f$ $=1200 / \sqrt{3}$ ). (c) Probability distribution $P\left(D^{2}\right)$ for different spring constants $f$ and the colloidal solid.

thermal, nonaffine noise smooths the discontinuities of the strain correlation functions. An examination of the neighborhood of particles with large $D^{2}$ reveals that this represents creation and annihilation of incipient defect-antidefect pairs producing a defect density field $[3,14]$ fluctuating about zero.

In summary, we have described $(T>0)$ strain-strain correlation functions for soft solids in $2 \mathrm{D}$. The correlation functions are shown to have a complex structure. The contribution of nonaffine displacements, which are essential and unavoidable, has important consequences for the nonlocal elastic response of soft solids. We believe that the strain correlation function may serve as a benchmark for characterizing soft crystals, being sensitive to the presence of defect pairs. Generalization of our results to higher dimensions and to less symmetric structures [20] is another direction for future studies.

Useful discussions with K. Binder, M. Rao, and S. Shenoy are gratefully acknowledged. This work and cooperation was funded by the Deutsche Forschungsgesellschaft (SFB TR6/ C4). Granting of computer time from HLRS, NIC, and SSP is gratefully acknowledged.
[1] P. M. Chaikin and T. C. Lubensky, Principles of Condensed Matter Physics (Cambridge University Press, Cambridge, UK, 1995).

[2] L. D. Landau and E. M. Lifshitz, Theory of Elasticity (Pergamon, Oxford, 1986).

[3] A. Buchel and J. P. Sethna, Phys. Rev. Lett. 77, 1520 (1996); see also T.-S. Lo A. Pomyalov, I. Procaccia, and J. Zylberg, e-print arXiv:0802.3404v1.

[4] A. Cemal Eringen, Nonlocal Continuum Field Theories (Springer, Heidelberg, 2002).

[5] T. A. Witten, J. Phys.: Condens. Matter 17, S1651 (2005).

[6] Soft Matter Physics, edited by M. Daoud and C. E. Williams (Springer, Heidelberg, 1999).

[7] P. N. Pusey, Science 309, 1198 (2005); A. Ramirez-Saito, C. Bechinger, and J. L. Arauz-Lara, Phys. Rev. E 74, 030401(R) (2006).

[8] S. Sengupta, P. Nielaba, M. Rao, and K. Binder, Phys. Rev. E 61, 1072 (2000); S. Sengupta, P. Nielaba, and K. Binder, ibid. 61, 6294 (2000).

[9] K. Zahn, A. Wille, G. Maret, S. Sengupta, and P. Nielaba, Phys. Rev. Lett. 90, 155506 (2003).

[10] P. Keim, G. Maret, U. Herz, and H. H. von Grünberg, Phys. Rev. Lett. 92, 215504 (2004).
[11] H. H. von Grünberg, P. Keim, K. Zahn, and G. Maret, Phys. Rev. Lett. 93, 255703 (2004); J. Zanghellini, P. Keim, and H. H. von Grünberg, J. Phys.: Condens. Matter 17, 3579 (2005).

[12] D. Reinke, H. Stark, H.-H. von Grünberg, Andrew B. Schofield, G. Maret, and U. Gasser, Phys. Rev. Lett. 98, 038301 (2007).

[13] X. H. Zheng and R. Grieve, Phys. Rev. B 73, 064205 (2006); I. Varga, H. Yamada, F. Kun, H.-G. Matuttis, and N. Ito, Phys. Rev. E 71, 051405 (2005).

[14] P. C. Martin, O. Parodi, and P. S. Pershan, Phys. Rev. A 6, 2401 (1972).

[15] M. Baus and R. Lovett, Phys. Rev. Lett. 65, 1781 (1990); Phys. Rev. A 44, 1211 (1991).

[16] M. L. Falk and J. S. Langer, Phys. Rev. E 57, 7192 (1998).

[17] D. Frenkel and B. Smit, Understanding Molecular Simulations, 2nd ed. (Academic Press, San Diego, 2002).

[18] K. Franzrahe et al. (unpublished).

[19] Unlike in [11] $\Gamma=150$ was calculated using $\chi_{M}$ determined by superconducting quantum interference device measurements, which have an error $\pm 10 \%$. An analysis according to [9] yields $\Gamma=175$.

[20] V. A. Froltsov, R. Blaak, C. N. Likos, and H. Löwen, Phys. Rev. E 68, 061406 (2003). 